\section{Species specificity of an adrenal androgen-mediated kill-switch triggered by p53 inactivation}

\author{
Jonathan W. Nyce \\ Advanced Canine Genetic Testing, \\ Collegeville, PA, USA
}

\section{Abstract}

Glucose-6-phosphate dehydrogenase (G6PD) is an oncoprotein that is regulated by the p53 tumor suppressor. Mutant p53 loses the ability to inhibit G6PD, and loss of G6PD control clearly plays a role in oncogenesis. The steroid hormone precursor dehydroepiandrosterone (DHEA) is an endogenous uncompetitive inhibitor of G6PD. In humans, and a few other species, the sulfated circulatory form of DHEA (DHEAS) is present at extremely high concentrations - much higher than can be accounted for by DHEA's function as a precursor to steroid hormones. Uncompetitive inhibition is extremely rare in natural systems because it is irreversible in the presence of high concentrations of substrate and inhibitor. What has gone unappreciated is that such uncompetitive inhibition can quickly lead to cell death when the target is an obligatory housekeeping gene such as G6PD. Cells with inactivated p53 not only lose control over G6PD, but also over hexokinase (HK), the enzyme that converts glucose into glucose-6-phosphate (G6P), the substrate of G6PD. Furthermore, loss of p53 function de-represses NFKB activity, resulting in the upregulation of steroid sulfatase (SS) which converts circulating DHEAS into active DHEA. We propose that inactivation of p53 rapidly elevates G6P and DHEA concentrations in affected cells, driving uncompetitive inhibition of G6PD to lethal irreversibility. In animals with circulating DHEAS, this kill-switch mechanism may prevent most cases of $\mathrm{p} 53$ inactivation from becoming tumorigenic. Tumors would thus represent instances in which this mechanism had not been triggered, but which might still be triggered by application of DHEA sufficient to uncompetitively inhibit tumor G6PD. To test this hypothesis, we performed a pilot study in which dogs with cardiac hemangiosarcoma were treated with high dose (HD) DHEA supplemented with isoprene precursors to maintain geranylation of Rac GTPase. Tumor regression and longevity observed in these dogs supported the concept that some tumors retain extraordinary sensitivity to uncompetitive inhibition by DHEA

\section{Introduction}

Primates are distinguished from most other animals by undergoing adrenarche, a developmental phase in which secretion of large amounts of DHEA heralds the onset of puberty. Adrenarche coincides with the development of the zona reticularis, a thin layer of tissue in the adrenal gland the sole function of which appears to be the synthesis of DHEA. The zona reticularis expresses high levels of CYP 17 required to synthesize DHEA from pregnenolone, and steroid sulfotransferase, which produces DHEA sulfate (DHEAS), the circulating form of DHEA. Due to the lack of $3 \beta$-hydroxysteroid dehydrogenase ( $3 \beta \mathrm{HSD})$, which is necessary to further metabolize DHEA to androstenedione and androstenediol - the proximate precursors of testosterone and estrone synthesis - DHEAS is secreted from the adrenal gland into the circulation. ${ }^{1}$ Circulating DHEAS is then transported into the testes and ovaries and a variety of peripheral tissues by the action of organic anion-transporting polypeptides (OATPs); thereafter, steroid sulfatase converts DHEAS into active DHEA. In sex steroid generating tissues, $3 \beta \mathrm{HSD}$ activity then converts DHEA to androstenedione and androstenediol; subsequently, aromatase, $17 \beta$-HSD and steroid $5 \alpha$-reductase further convert these ultimate precursors to $17 \beta$ estradiol, testosterone and $5 \alpha$-dihydrotestosterone, respectively. In the cytoplasm, the receptors for these sex steroids are bound to heat shock proteins that prevent their translocation into the nucleus. Testosterone and estrogen bind to their respective steroid receptors, effecting a conformational change in the receptors that causes heat shock proteins to release and diffuse away. The steroid bound receptor then transports into the nucleus, where it functions as a transcription factor. Following adrenarche, peripheral tissues such as the mammary gland in females and striatal muscle in males respond to activated steroid receptor, and cascades of gene expression changes bring about the secondary sexual characteristics associated with puberty. All during life in humans, the adrenal secretion of DHEAS and its conversion in peripheral tissues to DHEA by steroid sulfatase, permits tissue-specific synthesis of the appropriate intracellular amounts of DHEA required by cells. As noted above, in some tissues, DHEA is converted to sex steroids. $^{2}$ But the extremely high levels of circulating DHEAS in humans and some other long-lived primates far exceeds that required for sex steroid synthesis. Thus, the reason underlying such extraordinary levels
Correspondence: Jonathan W. Nyce, Advanced Canine Genetic Testing, 399 Arcola Road, P.O. Box 26219, Collegeville, PA 19426-3998, USA.

Tel: +1-484-255-1319.

E-mail: nyce.jonathan@acgt.us

Key words: Dehydroepiandrosterone, DHEA, p53 tumor suppressor, TP53, Peto's Paradox.

Acknowledgements: portions of this work were supported by the DeCecco Family Foundation. The diagnostic skills of Dr. Steven Liebl, DVM, and Dr. Nicolas Russell, DVM, ACVIM (Board certified cardiologist) are gratefully acknowledged. This work is dedicated with gratitude and affection to the memory of my mentors, Sydney Weinhouse, Peter Magee, and George Hitchings.

Conflict of interest: Professor Nyce is among listed inventors on several pending and issued patent applications on ACGT 011.

Received for publication: 1 May 2017

Revision received: 6 July 2017.

Accepted for publication: 18 July 2017.

This work is licensed under a Creative Commons Attribution 4.0 License (by-nc 4.0).

(C) Copyright J.W. Nyce, 2017

Licensee PAGEPress, Italy

Translational Medicine Reports 2017; 1:6773 doi:10.4081/tmr.6773

of circulating DHEAS remains a mystery. Circulating levels of DHEAS are higher in males than in females in humans and chimpanzees, and decline with age in humans and chimpanzees. ${ }^{3,4}$ Such age-related decline appears to be the result of a decrease in the number of functioning cells within the zona reticularis. This decline in circulating DHEA is associated temporally with an increase in risk in humans for such agerelated diseases as cancer. A corresponding age-related decline in immune function also occurs, known as immuno-senescence. DHEA is known to stimulate the innate immune system, ${ }^{5}$ and acts as a general counterbalance to anti-inflammatory glucocorticoids. ${ }^{6}$ As humans age, an imbalance in the Hypothalamic/Pituitary/Adrenal (HPA) axis occurs such that the DHEA to cortisol ratio dramatically decreases, contributing to immuno-senescence and degrading the immune surveillance that keeps most cancers at bay for the first two-thirds of the human lifespan. ${ }^{7}$ As we shall discuss below, high circulating levels of DHEAS may serve an even more fundamental anti-cancer role as a back-up for p53 tumor suppressor function, such that most instances of p53 inactivation may be prevented from leading 
to malignancy.

DHEA and DHEAS are also synthesized in the brain, where they function as neurosteroids. The neurogenic effects of DHEA and DHEAS were first reported by Roberts et al. who demonstrated that when dissociated rat brains growing in culture were exposed to either DHEA or DHEAS, prominent increases in the numbers of neurofilament-positive neurons and glial fibrillary acid protein-positive astrocytes were observed, with extensions of the processes of both types of cells. ${ }^{8}$ Compagnone and Mellon extended these results, demonstrating that the developmentally regulated, region-specific expression of CYP17 in the rat embryo is involved in brain development. Thus, CYP17 is expressed in neurons of the cortical sub-plate, a region involved in the guiding of thalamic fibers to their cortical targets. Compagnone and Mellon demonstrated that, in cultures of neocortical neurons of embryonic 16.5 day rats, DHEA selectively increased the length of neurites containing the axonal marker Tau-1, and the incidence of varicosities and basket-like process formations, whereas DHEAS selectively increased the length of neurites containing the dendritic marker MAP-2. ${ }^{9}$ In humans, also, DHEA appears to shape amygdala-dependent cortical plasticity as measured by functional magnetic resonance imaging studies. ${ }^{10,11}$

\section{DHEA supplementation in clini- cal and epidemiological studies}

As described elegantly by Labrie and Labrie, while synthesis of androgens and estrogens have an evolutionary history that is several hundred million years old, the advent of high levels of circulating DHEAS is a recent evolutionary event, originating primarily in primates and a very few other mammalian species about 20 million years ago. ${ }^{12}$ DHEAS can circulate in high concentrations systemically because it is safe, essentially inactive as a steroid, and yet can be rapidly transformed into DHEA and then into steroids on an as needed basis in various peripheral tissues. This evolutionary advance has been mimicked in certain treatments for menopausal women in whom circulating DHEAS levels are sub-normal. For example, the U.S. Food and Drug Administration has recently approved an intravaginal suppository form of DHEA for the treatment of women experiencing dyspareunia, a symptom of vulvar and vaginal atrophy due to menopause. ${ }^{13-}$ ${ }^{15}$ In this way, providing local DHEA at the site where it is needed overcomes the subnormal levels of DHEAS present in some post- menopausal women, and avoids the potential risks of estrogen exposure to other tissues.

Clinical supplementation with oral DHEA has also been shown to improve fertilization results in women with Diminished Ovarian Reserve (DOR). In a double-blind randomized, placebo-controlled clinical trial, Zhang and colleagues demonstrated that DHEA administration significantly improved embryo score in infertility patients with DOR, as compared to controls ${ }^{16}$ (but see also Yeung et al. ${ }^{17}$ ). In a double-blind, placebocontrolled clinical trial of the use of oral DHEA to modify drug abuse behavior, DHEA administration had a long-lasting preventive effect on relapse to drug use. In a 16month follow-up, relapse rates of DHEAtreated patients were one-third those of the placebo-treated group. ${ }^{18}$ Also, it appears that Selective Serotonin Uptake Inhibitors (SSRIs) used in major depression may function in relation to endogenous levels of serum DHEA. Thus, Hough et al. found that serum DHEA levels were positively correlated with response to SSRI treatment in patients with major depression. ${ }^{19}$ Similarly, Schmidt and colleagues, at the National Institutes of Health Clinic in Bethesda, Maryland, used a double-blind, randomized, placebo-controlled cross-over study of DHEA $v s$ placebo in patients with midlife-onset depression, and observed a significant improvement in patients receiving oral DHEA for six weeks. ${ }^{20}$ Dumas de la Roque and colleagues demonstrated a significant improvement in pulmonary hypertension in patients with chronic obstructive pulmonary disease (COPD) treated with oral DHEA. Pulmonary hemodynamics and 6-minute walk test were also improved. ${ }^{21}$

Taken together, these data suggest that endogenous DHEA and DHEAS serve important functions both in the brain and in peripheral tissues of humans, and that pharmacological administration of DHEA can correct certain aspects of aberrant physiology both in the brain and in peripheral tissues. However, all of these uses and known functions of DHEA relate to its role as a precursor for steroid hormone synthesis, or as an immune modulator. Neither of these roles can account for the extremely high levels of circulating DHEAS that exist in humans, and neither address the most unusual and least explained feature of DHEA - its function as an uncompetitive inhibitor of G6PD.

\section{DHEA, p53 and Glucose-6-phos- phate dehydrogenase}

One of the most fascinating things about DHEA is that it is an endogenous, uncompetitive inhibitor of the enzyme G6PD. G6PD is gaining increasing recognition as an oncoprotein, as demonstrated by the fact that all that is necessary to transform a non-tumorigenic cell into one capable of forming tumors in vivo is to insert additional active copies of G6PD into it. ${ }^{22}$ G6PD is also upregulated in most, perhaps all tumors, and appears to be a key determinant of the Warburg effect, a hallmark of the neoplastic state. ${ }^{23}$ G6PD is the rate-limiting enzyme of the Pentose Phosphate Pathway (PPP), the main source of cellular NADPH. Transformed cells require excessive amounts of NADPH to fuel their enhanced rate of growth, to maintain glutathione and thioredoxin in the reduced state necessary to detoxify reactive oxygen species (ROS), and for the epigenetic inactivation of tumor suppressor genes using S-adenosylmethionine (SAM) as the methyl donor. Perhaps the single most important piece of evidence implicating G6PD as an oncoprotein critical to the transformed state is the fact that the p53 tumor suppressor, the most frequently mutated locus in human and animal cancer, has as one of its main functions the inhibition of this enzyme. Mutant p53 proteins lose their ability to inhibit G6PD, removing intracellular NADPH availability as a constraint upon tumor growth. ${ }^{24}$ The uncompetitive type of inhibition of G6PD mediated by DHEA is extremely rare in nature. Unlike a competitive inhibitor, an uncompetitive inhibitor can bind only to the enzyme substrate complex (E-S) because E$\mathrm{S}$ binding flexes the protein, creating the binding site for such an inhibitor. This type of enzyme inhibition can exert extreme effects upon metabolic intermediates compared to other forms of inhibition. Thus, any type of inhibition will tend to increase the concentration of substrate. With competitive inhibition, this increasing amount of substrate will eventually out compete the inhibitor, returning the system toward normal. But with uncompetitive inhibition, the increasing concentration of substrate creates a feed-forward inhibition such that an uncompetitive inhibitor cannot be overcome, as both Vmax and $K m$ are equally reduced..$^{25}$ This is modeled by the equation:

$$
V=\frac{V_{\max }^{a p p}[S]}{K_{m}^{a p p}+[S]}
$$

where $V_{\max }^{a p p}$ is the apparent $V_{\max }$ given by:

$$
V_{\max }^{a p p}=\frac{V_{\max }}{1+\frac{[I]}{K_{i}}}
$$


And $K_{m}^{a p p}$ is the apparent $K_{m}$ given by:

$$
K_{m}^{a p p}=\frac{K_{m}}{1+\frac{[I]}{\left[K_{i}\right]}}
$$

We propose that the uncompetitive inhibition kinetics of DHEA enable it to rapidly degrade G6PD activity in cells in which p53 function has been compromised, providing a kill-switch mechanism that induces cell death in most instances of p53 inactivation. In support of this proposal, consider these facts. Glucose uptake is regulated by $\mathrm{p} 53$ by inhibiting glucose transport proteins. ${ }^{26}$ Cells with mutant p53 thus lose the capacity to regulate glucose uptake, and internalized glucose is rapidly phosphorylated to Glucose-6-phosphate (G6P) by hexokinase (HK) enzymes. P53 also regulates HK activity by enhancing the maturation of miR-143, a potent inhibitor of HK expression. ${ }^{27,28}$ In cells in which p53 has been inactivated, G6P levels will rise because of loss of miR-143 regulation of $\mathrm{HK}$, and most or all G6PD enzymes will consequently be sequestered within an E-S complex sensitive to uncompetitive inhibition by DHEA. The kill-switch mechanism that we propose depends upon simultaneous rapid upregulation of DHEA concentrations upon p53 inactivation in a cell. Under normal circumstances, p53 inhibits $\mathrm{NF \kappa B},{ }^{29,30}$ a proinflammatory mediator that activates steroid sulfatase, ${ }^{31}$ the enzyme that converts circulating DHEAS to active DHEA in peripheral tissues. In cells with inactivated p53, loss of p53-mediated NFKB inhibition will result in upregulation of steroid sulfatase, increasing intracellular DHEA concentrations which will accelerate the feed forward uncompetitive inhibition of G6PD. Intracellular DHEA potentiates this process still further by inducing GLUT4 and HK activity, ${ }^{32}$ raising G6P levels, and by enabling G6P to accumulate by inhibiting Glucose-6-phosphatase. ${ }^{33}$ This would represent a sort of dead man switch for p53 in which its inactivation activates its back-up, DHEA. In cells in which the primary tumorigenic lesion is inactivation of $\mathrm{p} 53$, by its uncompetitive kinetics DHEA may be able to drive the feed forward inhibition of G6PD so rapidly and potently that cell death ensues (Figure 1). This back-up mechanism depends upon there being circulating DHEAS that can be accessed by cells anywhere in the body that have experienced p53 inactivation (Figure 2). The ubiquitous expression of OATPs ${ }^{34}$ and steroid sulfatase $^{35}$ in virtually all tissues of the human body, and of a much more limited expres- sion of $3 \beta-H S D$, aromatase, $17 \beta-H S D$ and steroid $5 \alpha$-reductase, point to a nonsteroidogenic role for circulating DHEAS, such as the one we are proposing, in addition to its function as a steroid hormone precursor. Further support for this idea comes from the fact that serum DHEAS levels decline with age in human males at a rate that is almost three times faster than that for testosterone. ${ }^{36}$ Clearly, if circulating DHEAS was limited to its role as a precursor for sex steroids, its levels would parallel those of the steroids for which it acts as precursor. Also consider that, in contrast to castrated dogs which have a significantly increased risk of cancer, castrated male humans do not have an elevated risk of cancer. ${ }^{37}$ While the testes are the source of
DHEA in dogs (see below), in humans DHEA is synthesized and secreted by the zona reticularis in the adrenal glands. Castration thus prevents DHEA synthesis in the dog, but not the human.

\section{Of mice and men}

While systemic DHEA secreted by the adrenals plays a critical role in the development and physiological homeostasis of humans and some other primates, ${ }^{38,39}$ rodents such as rats and mice have not evolved a zona reticularis, do not express CYP17 in adrenal or gonadal tissue, and therefore have no circulating DHEAS. ${ }^{40}$

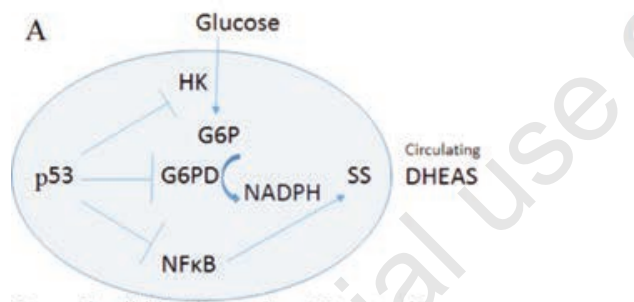

Normal cell kinetics under p 53 control

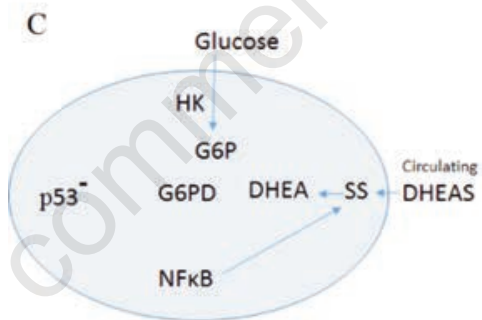

p53 inactivation also activates DHEA "kill-switch"

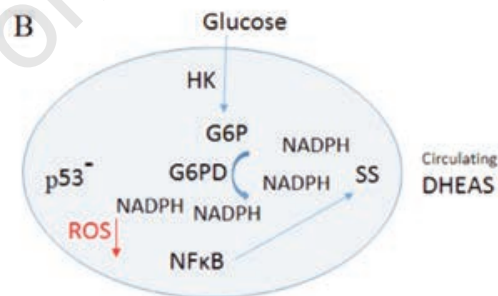

p53 mutation activates G6PD and HK: Sufficient to initiate and maintain tumorigenesis

D

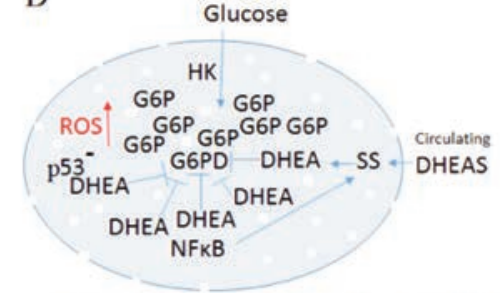

Uncompetitive inhibition is irreversible and lethal with high substrate and inhibitor concentrations

Figure 1. Mechanism of dehydroepiandrosterone (DHEA) kill-switch in cells with p53 mutation. A) Under normal circumstances, p53 inhibits numerous enzymes and transcription factors, three of which are Hexokinase (HK), Glucose-6-phosphate dehydrogenase (G6PD), and Nuclear Factor $K$ B (NFKB). NFKB is a potent stimulator of Steroid Sulfatase (SS) activity, the enzyme that activates inactive, circulating DHEA sulfate (DHEAS) into active DHEA. B) When TP53 is inactivated, it loses the capacity to inhibit HK and G6PD. Under such circumstances, HK supplies increased amounts of Glucose6-phosphate (G6P), the substrate of G6PD. G6PD, released from TP53-mediated repression, produces the excessive amounts of NADPH required by transformed cells for reductive biosynthesis such as nucleotide synthesis; for the $S$-adenosylmethionine that acts as methyl donor in the DNA hypermethylation reactions that are a hallmark of the malignant state; for the mevalonate pathway products that target oncoproteins such as Ras to their intracellular compartment; and for the maintenance of redox proteins in their reduced state so that reactive oxygen species (ROS) can be kept under control. Loss of G6PD control is sufficient to render a non-tumorigenic cell tumorigenic. C) In humans and other animals with circulating DHEAS, the release of NFKB from p53 control activates SS. Circulating DHEAS is de-sulfated into active DHEA, an uncompetitive inhibitor of G6PD. D) In the presence of de-repressed HK and activated SS, G6P and DHEA levels increase, irreversibly inhibiting G6PD, and depleting NADPH such that control of ROS becomes impossible. Activation of this kill-switch in cells with inactivated TP53 prevents most TP53 inactivation events from becoming tumorigenic. This killswitch mechanism can be active only in animals with circulating DHEAS. 
Rats and mice do employ DHEA as a neurosteroid, but its use in such rodents appears to be limited to brain development and physiology. These common laboratory species have not evolved adrenal androgens, and thus no mechanism employing systemic DHEAS can exist in them. Despite this limitation of common laboratory rodents as model systems for DHEA in humans, many studies have been performed demonstrating an anticancer effect for DHEA in chemically-induced ${ }^{41}$ and gene knock out-mediated rodent cancers. ${ }^{42}$ One of the models most commonly used to investigate cancer mechanisms has been the p53 knock out mouse. Such mice are extremely cancer prone, just as humans with genetic mutation of TP53 (Li-Fraumeni syndrome) are extremely cancer prone. This murine mimic of Li-Fraumeni syndrome, by exactly the same mechanism - p53 mutation - has given the research community a sense of comfort that the p53 knock out mouse offers a reasonable model system with direct application to human cancer. However, we believe that this comfort with the p53 knock out mouse as a model for human cancer is not well founded; that in fact, the p53 systems of man and mouse are extremely dissimilar, for the reason that mice do not have circulating DHEAS.

In addition to circulating DHEAS, species-specific evolutionary modifications to additional enzyme systems would be required to enable the adrenal androgenmediated kill switch to operate. For example, Glucose-6-phosphatase catalytic subunit (G6PC) metabolizes G6P to glucose and inorganic phosphate. Its metabolism of G6P thus prevents the formation of an ES complex between G6PD and G6P, which would not permit the function of the described kill switch mechanism requiring uncompetitive inhibition of G6PD. If the adrenal androgen-mediated kill switch evolved in humans but not mice, is there evidence for speciesspecific evolution of G6PC? Peroxisome Proliferator Activator Receptor Gamma Coactivator-1 $\alpha$ (PGC-1 $\alpha$ ), an important regulator of metabolism, markedly stimulates mouse G6PC activity via Hepatic nuclear factor- $4 \alpha$ (HNF-4 $\alpha)$, a member of the steroid/thyroid hormone receptor superfamily. HNF- $4 \alpha$ binds to an element located between -76 and -64 in the mouse G6PC promoter. It is therefore of interest that even though this -76 to $-64 \mathrm{HNF}-4 \alpha$ binding site is perfectly conserved in the human G6PC promoter, PGC-1 $\alpha$ does not stimulate human G6PC activity. Schilling and colleagues demonstrated that this species-specific difference could be explained by a $3 \mathrm{bp}$ sequence variation, located immediately adjacent to a consensus nuclear hormone receptor half-site that is perfectly conserved between the mouse and human G6PC promoters. ${ }^{43}$ With gel retardation experiments, Schilling and her colleagues demonstrated that this $3 \mathrm{bp}$ variation in the human G6PC promoter extinguishes HNF- $4 \alpha$ binding to the half-site. DHEA is a potent peroxisome proliferator and a known inducer of PGC$1 \alpha^{44}$ In mice, DHEA would therefore stimulate G6PC activity and thereby induce the elimination of G6P substrate, preventing irreversible uncompetitive inhibition of G6PD. In humans, the 3 bp variation in the G6PC promoter permits the adrenal androgen-mediated kill switch to function by enabling the accumulation of G6P substrate in the presence of DHEA. The discovery of this $3 \mathrm{bp}$ difference between human and mouse G6PC promoters that controls G6P substrate accumulation appears to offer an additional window on the species-specific evolution of the adrenal androgen-mediated kill switch in man. It also explains why administration of DHEA to p53-/- mice is not toxic.

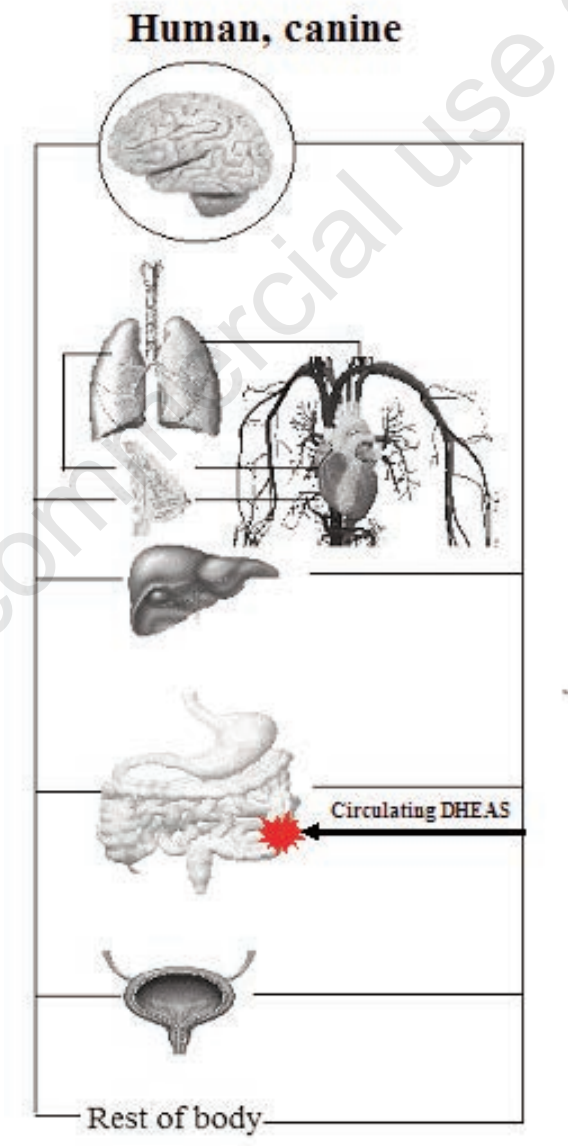

\section{Peto's paradox}

Richard Peto, a statistical epidemiologist at the University of Oxford, demonstrated mathematically that, on a cell for cell basis, human cells in vivo appear to be approximately $10^{9}$ more resistant to carcinogenesis than are mouse cells. ${ }^{45,46}$ As Peto wrote:

A man has 1000 times as many cells as a mouse... and we usually live at least 30 times as long as mice. Exposure of two similar organisms to risk of carcinoma, one for 30 times as long as the other, would give perhaps $30^{4}$ or $30^{6}$ times the risk of carcinoma induction per epithelial cell. However, it seems that, in the wild, the probabilities of carcinoma induction in mice and in men are not vastly different. Are our stem cells real$l y$, then, a billion or a trillion times more "cancer-proof" than murine stem cells? This is biologically pretty implausible; if human DNA is no more resistant to mutage-

\section{Laboratory mice, rats}
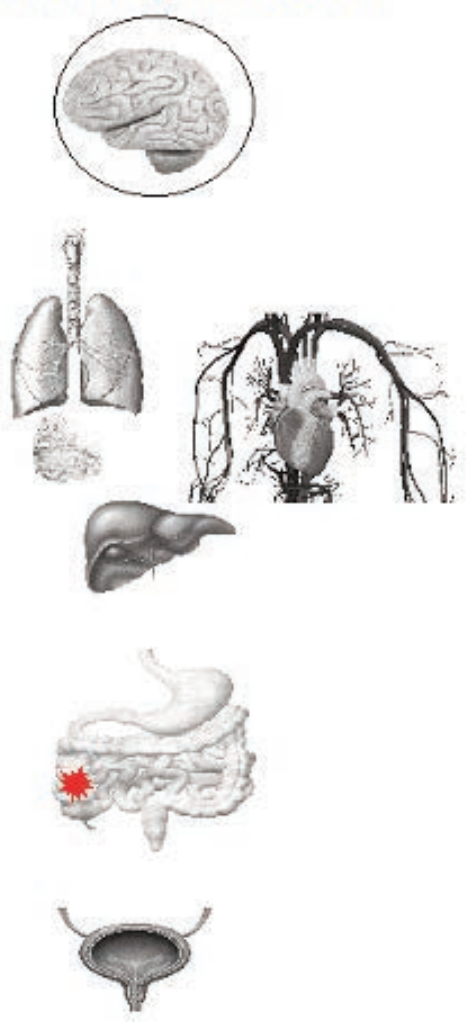

Figure 2. Circulating dehydroepiandrosterones (DHEAS). Whereas humans and some animals, such as dogs, have circulating DHEAS (left) available for conversion to DHEA in cells that have undergone p53 inactivation, DHEA and DHEAS are limited to the brain in laboratory mice and rats (right). Circulating DHEAS and activation of STS and HK in cells undergoing p53 inactivation (red symbol) enables the uncompetitive G6PD inhibition kinetics of DHEA to become irreversible, inducing cell death and thereby preventing tumorigenesis. If DHEA is part of such a kill-switch mechanism in cells with inactivated p53, such mechanism will not be operative in common laboratory animals such as mice and rats, bringing into question their relevance to human cancer. STS, steroid sulfatase. 
nesis in vitro than mouse DNA, why don't we all die of multiple carcinomas at an early age?

The answer to Peto's Paradox may be that humans, but not mice, have circulating DHEAS and the appropriate enzymes in all tissues of the body to convert that circulating DHEAS into DHEA in the event of p53 inactivation. Once converted, the uncompetitive kinetics of DHEA's inhibition of G6PD rapidly becomes irreversible in the presence of high intracellular substrate concentrations of G6P caused by de-repression of HK, and of high intracellular concentrations of DHEA inhibitor caused by activation of steroid sulfatase (Figure 1). Because G6PD activity supplies the NADPH reducing power required to detoxify ROS, in the absence of G6PD activity ROS rapidly increase to lethal levels. The beauty of this evolved mechanism is that it will only be activated in cells that have experienced p53 inactivation. This explains why humans, with a thousand times more cells than a mouse, and a life span 30 times longer than that of a mouse, experience no increased risk of dying of cancer compared to a mouse. It also explains why there would exist an uncompetitive inhibitor of a critical housekeeping enzyme like G6PD. So to answer Peto's paradox directly, yes, human stem cells are a billion times more cancer-proof than murine stem cells because human stem cells have a DHEA back-up, should they experience inactivation of their p53, and murine stem cells do not; and the reason that human DNA is no more resistant to mutagenesis in vitro than is mouse DNA, is that in the in vitro setting, circulating DHEAS has been removed from the equation. In a sense, in the in vitro setting, human cells have been de-evolved into the equivalent of mouse cells.

There are, of course, animals larger than man and equally long-lived. Such animals, e.g., the elephant, appear to have adopted a different strategy to reduce the cancer risk associated with such enormous body mass, and its vast quantity of cells. Elephants have multiple copies of p53 in their genomes. ${ }^{47,48}$ They thus use the same p53 system, but in over-drive, to limit carcinogenic events in their massive bodies. This begs the question of why primates, particularly man, went a different route, evolving circulating DHEA instead of increasing copy number of p53. It may be that increasing p53 copy number is a strategy incompatible with some aspect of human evolution. Alternatively, circulating DHEAS may have opened doors to human evolution in addition to those that we have discussed. For example, because DHEA acts as an anti-glucocorticoid, inhibiting the HPA axis-mediated fight or flight response, it has been suggested that the high, primate-specif- ic levels of DHEA of early humans may have increased social interaction with nonfamiliar individuals, contributing to the ability of our species to commune together in ever larger groups. ${ }^{38}$

If our hypothesis is correct that DHEA acts as a kill-switch for cells that have experienced p53 inactivation, then experiments conducted in p53 knockout mice may have little or nothing to do with human cancer. It is thus an unfortunate accident of history that almost all basic science data utilizing DHEA administration in cancer, and virtually all of the preclinical data exploring the role of p53 in carcinogenesis, have been obtained in species in which systemic DHEA and its function as a back-up for p53 do not exist. Without such evolutionary context, laboratory mice and rats and the tumors that can be made to occur in them by many different means appear to represent exceptionally poor windows on the potential of DHEA to modulate cancer in humans, or to tell us very much that is relevant regarding the role of p53 in human cancer.

\section{Canines}

Dogs do not duplicate primate adrenarche, but they appear to have a homologue of it. ${ }^{49,50}$ Thus, juvenile canines have low circulating DHEAS levels, which rise and create peak concentrations prior to puberty, just as in humans and chimpanzees; and male dogs have higher levels of circulating DHEAS than females, just as in humans and chimpanzees..$^{51,52}$ Similarly, canines appear to have a functioning adrenal zona reticularis capable of producing DHEA, although certainly not at the levels produced in primates. ${ }^{53}$ In dogs, as in humans, levels of circulating DHEAS decline with age, with the additional fact that such decline occurs earlier in ovariectomized canines. ${ }^{54,55}$ Unlike humans, virtually all the DHEA in male dogs appears to be synthesized in the testes, such that castration leads to a decrease in systemic DHEAS to negligible levels. ${ }^{50}$ Recent evidence indicates that castrated male dogs and ovariectomized female dogs are at dramatically increased risk of developing a variety of different cancers. ${ }^{55}$ This has led our laboratory to suggest that this increased cancer risk in neutered dogs is due to the loss of circulating DHEAS and the anti-cancer properties that it would otherwise effect. ${ }^{57}$ Taken together, these data sets indicate that, unlike common laboratory rats and mice, circulating DHEAS has an evolutionary context in canines. Dogs thus offer a model system for the study of p53 and DHEA that common laboratory rodents cannot approach. As Sa and colleagues have recently demonstrated, additional aspects of canine physiology make them preferred models over many other species for their similarity to man. ${ }^{58}$ As we will discuss below, our discovery that a subpopulation of canine tumors exists that is hypersensitive to G6PD inhibition offers additional support for a p53 back-up role for DHEA in animals with circulating DHEAS. As in humans, it may have been the case that circulating DHEAS in dogs served multiple functions. We suggest that DHEA's inhibition of the fight-or-flight response may have contributed to the domestication of the dog by selecting for wolves tolerant of human contact.

We reasoned that if DHEA did act as a kill-switch in cells in which p53 had been inactivated in the initial stage of carcinogenesis, then there might exist a subset of tumors in which this mechanism had failed to trigger (hence the tumor growth), but which might still be able to be triggered if exogenous DHEA were added to the system sufficient to induce G6PD inhibition within the tumor. This mechanism would not be operative in species like laboratory rodents which do not have circulating DHEAS. We therefore identified owners of dogs with various spontaneous tumors who were willing to participate in this research program. After obtaining informed consent, we treated dogs with histologically confirmed spontaneous tumors with oral HD DHEA in divided daily doses.

\section{Autoinflammatory reaction occurring in dogs treated with high dose DHEA}

Our original protocol consisted of HD DHEA $(60 \mathrm{mg} / \mathrm{kg})$ and ubiquinone $(0.2$ $\mathrm{mg} / \mathrm{kg}$ ). In the first dogs treated with this protocol, we observed an autoinflammatory reaction that closely resembled the human autosomal recessive disease Mevalonate Kinase Deficiency (MKD).$^{59,60}$ Both our initial canines and people with MKD experienced recurrent febrile episodes, arthralgia, apparent myalgia, severe skin rashes and apthous ulceration of mucocutaneous tissues, especially about the eyes and mouth. ${ }^{61}$ In a series of in vitro studies we had previously demonstrated that DHEA, via depletion of NADPH, inhibits HMG Co A Reductase (HMGCR) and all downstream enzymes, including mevalonate kinase, ${ }^{62}$ and blocked the isoprenylation of the Ras oncoprotein. ${ }^{63}$ Both MKD and the autoinflammatory reaction in dogs resulting from 
HD DHEA appear to be due to induction of IL1 $\beta$ secretion caused by inhibition of the isoprenylation of the Rac small GTPase. ${ }^{64}$ As we had demonstrated in vitro, and others have demonstrated in animal models of MKD, ${ }^{65,66}$ reconstitution of protein isoprenylation using terpenes $(\mathrm{T})$, prevents this autoinflammatory reaction from occurring.

One important feature of our finding that HD DHEA treatment in dogs leads to the development of an autoinflammatory reaction that closely resembles human MKD, and that it can largely be prevented via reconstitution with terpenes capable of enabling protein isoprenylation in a manner analogous to animal models of $\mathrm{MKD}$, is that it provides strong evidence that high dose (HD) DHEA depletes NADPH on a systemic level in vivo. It is of interest that extremely high doses of DHEA have been administered to rats and mice without any induction of such an autoinflammatory reaction. This is probably due to the fact that systemic DHEAS has not evolved in common laboratory rodents, and so they cannot and do not respond in the same fashion to the simulation of systemic DHEAS as dogs, and presumably humans, do.

\section{Hypersensitivity to G6PD inhibi- tion in a subset of canine tumors}

We identified a subpopulation of canine tumors that responded to HD DHEA/T by undergoing complete and durable regression, without the induction of the autoinflammatory reaction. Only a small fraction of most tumor types (e.g., Figure 3), responded to HD DHEA/T with complete and durable regression. This was probably due to the fact that most tumors had advanced to a stage in which many followon mutations in addition to p53 mutation had occurred, interfering with the ability of DHEA to trigger irreversible uncompetitive inhibition of G6PD. However, canine cardiac hemangiosarcoma $(\mathrm{CCH})$ was a clear exception. In four consecutive dogs with $\mathrm{CCH}$ (hemorrhagic pericardial effusion; right atrial mass), ACGT 011 induced complete and durable tumor regression. Generating survival curves using the product limit method of Kaplan and Meier, ${ }^{67}$ we compared our longevity results to those obtained by Yamamoto et al., the group that has published the best results to date. ${ }^{68} \mathrm{We}$ then individually compared survival curves for G6PD inhibition against each of the Yamamoto treatment groups using the logrank test of Mantel-Cox, generating the statistical data shown in Table 1. Dogs with $\mathrm{CCH}$ treated by inhibition of G6PD had complete or near-complete resolution of their tumor (Figure 4), and dramatically extended longevity compared to the dogs in the Yamamoto study. Thus, in the Yamamoto study, untreated dogs with $\mathrm{CCH}$ had a median survival (MS) of 7.1 days; dogs treated with extensive chemotherapy (cyclophosphamide, vincristine, doxorubicin) had a MS of 27 days; dogs treated with pericardectomy to remove tumor had a MS of 86 days; and dogs treated with a combination of chemotherapy and pericardectomy had a MS of 189 days. This compared to a MS of 1112 days for our dogs treated with G6PD inhibition, with two of

Table 1. Log-rank (Mantel-Cox) comparison of survival curves of each group following Yamamoto et al. ${ }^{63}$ with G6PD inhibition data. Hazard ratios (Yamamoto/G6PD inhibition) were computed with GraphPad Prism software Version 7.03.

\begin{tabular}{lccccc} 
Group & $\begin{array}{c}\text { Median survival } \\
\text { (Days) }\end{array}$ & Havard ratio & Chi square & df & P value \\
Untreated & 7.1 & $10.86 / 0.09204$ & 9.5 & 1 & 0.0021 \\
Chemotherapy & 27 & $5.32 / 0.1879$ & 12.72 & 1 & 0.0004 \\
\hline Surgery & 86 & $7.26 / 0.1377$ & 10.29 & 1 & 0.0013 \\
Chem + Surg & 189 & $14.2 / 0.07041$ & 7.91 & 1 & 0.0049 \\
\hline G6PD Inhib & $1112^{*}$ & & & & \\
\hline
\end{tabular}

df, degree of freedom. *Since two G6PD inhibition dogs are still alive at the time of this writing (at 840 and 1500 days), their survival data was entered as censured data points.

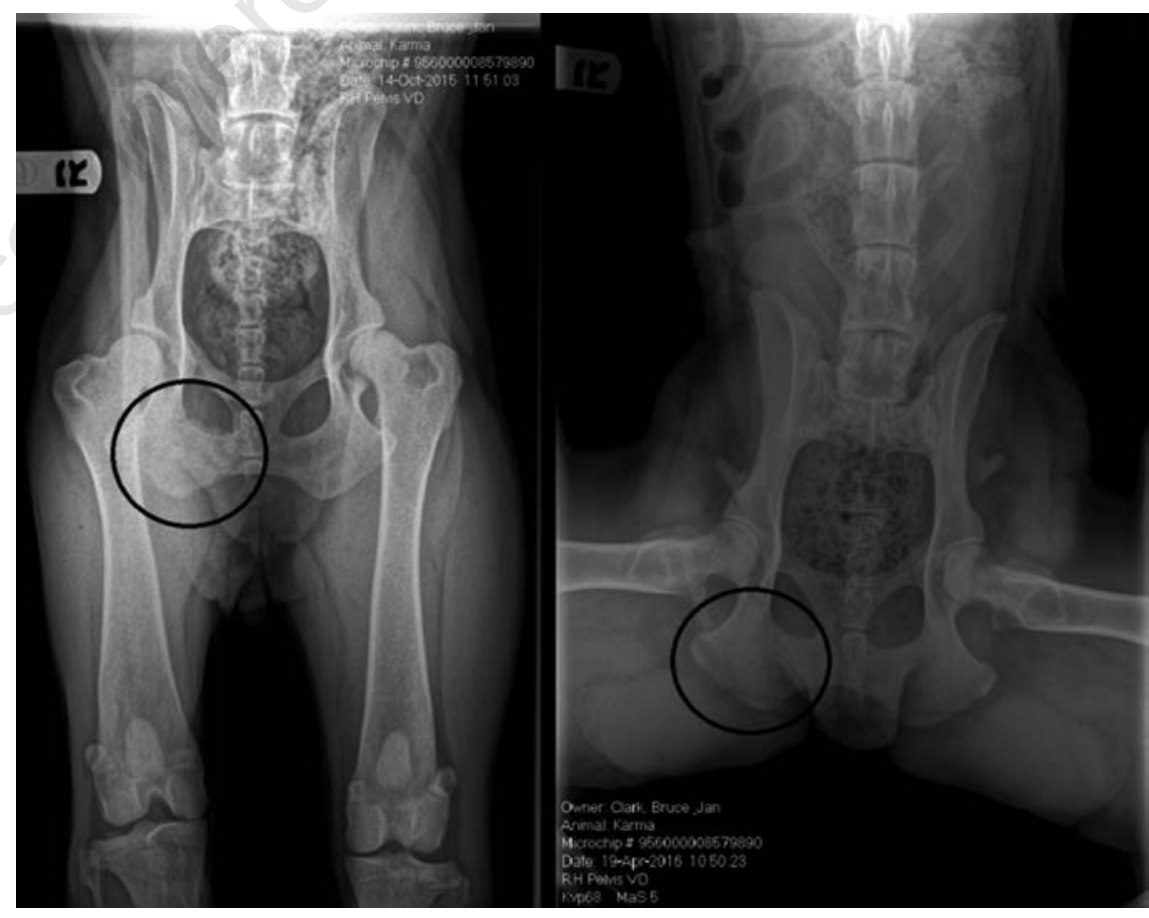

Figure 3. Left, histologically verified soft tissue sarcoma creating extreme lameness in female Doberman KC before HD dehydroepiandrosterone (DHEA). Right, complete regression of tumor after HD DHEA. KC conceived and delivered a litter of five healthy puppies while being treated with ACGT 011, demonstrating that properly reconstituted HD DHEA is not cytotoxic to the developing fetus. $\mathrm{KC}$ remains alive and well with unfettered mobility and no evidence of recurrence 21 months after cessation of treatment; 25 months after diagnosis. 
of HD DHEA in dogs with CH. We had previously demonstrated in vitro that DHEA inhibits HMGCR indirectly, via NADPH cofactor depletion, ${ }^{62}$ with consequent inhibition of protein isoprenylation. ${ }^{63}$ In the first dogs studied we observed a severe autoinflammatory reaction closely resembling MKD in humans with an inactive mevalonate kinase gene. There appear to be no published accounts of such an MKD-like inflammatory reaction occurring in normal laboratory rats or mice, probably because, unlike humans and canines, these species do not have circulating DHEAS, and therefore lack an evolutionary context to respond to exogenous DHEA in the same way that dogs (and presumably humans) do. When we administered properly reconstituted HD DHEA/T (ACGT 011) to four consecutive dogs with $\mathrm{CH}$, we observed durable tumor regression and survival times an order of magnitude greater than the best published results in canine $\mathrm{CH}$ to date. These results provide additional evidence that HD DHEA/T depletes NADPH in vivo in a manner analogous to our previous in vitro experiments. Other tumor types also demonstrated subpopulations that were hypersensitive to G6PD inhibition, but at much lower frequency than $\mathrm{CH}$. The extreme sensitivity to HD DHEA demonstrated by $\mathrm{CH}$ may relate to the special role that ROS and NOS play in endothelial cell physiology. For example, the redox cofactor tetrahydrobiopterin (BH4) requires multiple NADPH-dependent steps in its biosynthesis and is therefore depleted with HD DHEA treatment. BH4 depletion leads to uncoupling of endothelial nitric oxide synthase, resulting in the production of superoxide $\left(\mathrm{O} 2{ }^{-}\right){ }^{69}$ Superoxide further oxidizes BH4, creating a feed forward increase in ROS. This may occur preferentially in the endothelial cells giving rise to $\mathrm{CH}$, making them hypersensitive to further increase in ROS resulting from HD G6PD. LopezMarure's group has shown that DHEA inhibits proliferation of human endothelial cells in vitro in an androgen and estrogen receptor-independent manner, providing support for this model. ${ }^{70}$

It is also important to consider other potential sources of NADPH in endothelial and other cell types. Isocitrate Dehydrogenase (IDH), for example, may be capable of producing sufficient NADPH to rescue cells from ROS-mediated cell death. It is thus possible that tumors that do not respond to HD DHEA may have already undergone selection for elevated IDH expression. IDH has been observed to be over-expressed in certain tumors. ${ }^{71,72}$ Alternatively, it may be that canine tumors that do respond to HD DHEA with durable tumor regression, such as $\mathrm{CH}$, may harbor IDH mutations sensitizing them to NADPH depletion. Thus, IDH mutations have been shown to create a gain of function such that $\alpha$-ketoglutarate is further metabolized to the oncometabolite 2-hydroxyglutarate in a reaction that consumes, rather than produces, NADPH (Figure 5). ${ }^{73}$ Tumors with IDH mutations causing consumption rather than production of NADPH may be particularly sensitive to HD DHEA. Whatever the reason underlying the extreme hypersensitivity of canine $\mathrm{CH}$ to HD DHEA, cardiac angiosarcoma is believed to represent the identical disease in humans. Like $\mathrm{CH}$ in dogs, cardiac angiosarcoma in humans is a deadly form of cancer that strikes a younger patient population than most other cancers. ${ }^{74}$ It will be important to determine if our results in canine $\mathrm{CH}$ will translate directly to human cardiac angiosarcoma. If
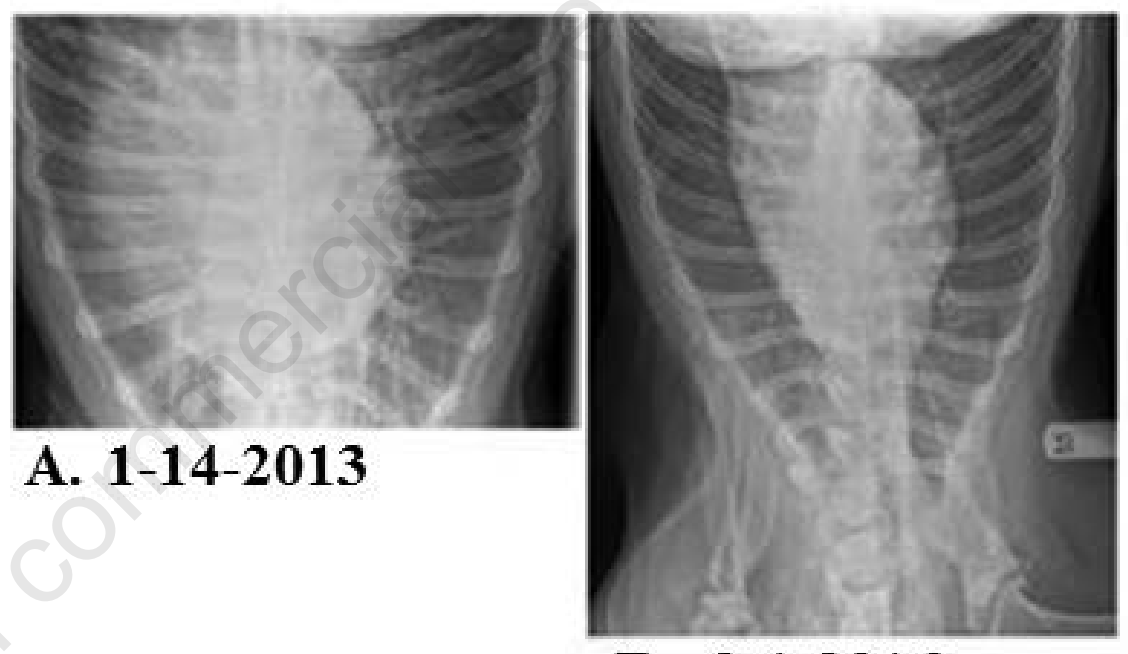

B. 2-1-2016

Figure 4. Cardiac hemangiosarcoma occurring in a Labrador Retriever (GC) diagnosed by a certified veterinary cardiologist. GC presented with hemorrhagic pericardial effusion and massive right atrial tumor mass (A). After ACGT 011, this tumor mass underwent complete regression (B).<smiles>O=C([O-])CC(C(=O)[O-])C(=O)[O-]</smiles>

Isocitrate

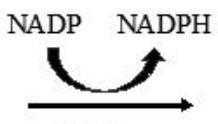

$\mathrm{IDH}$
Figure 5. Tumors that have undergone selection for high expression of wild type IDH may be resistant to uncompetitive inhibition of G6PD by DHEA because the requirement for NADPH to maintain glutathione and thioredoxin in a reduced state, and thereby control ROS, is met. Tumors which have IDH mutations that consume rather than produce NADPH may be particularly sensitive to G6PD inhibition by DHEA. 
lars spent since the discovery of the p53 tumor suppressor gene, the average increase in survival for most cancer patients over this period of time has improved by a mere three months. ${ }^{77}$ An example of this relative failure to improve survival is lung cancer. A study of 971 lung cancer patients diagnosed and treated in 2002, compared to 927 patients diagnosed and treated in 1985, showed an improvement over this 17-year period of only 33 days. ${ }^{78}$ Ten-year survival in lung cancer, currently at 5\% in developed countries, has remained relatively unchanged for more than forty years. ${ }^{79}$ Even with the recent break throughs in immune checkpoint inhibition in some patients with non-small cell lung cancer, the average improvement in patient survival using this new technology is generally less than four months. ${ }^{80}$ It is unsettling to think that, because of a heretofore unrecognized relationship between circulating DHEAS and p53 function, the use of model systems that do not translate to man may have contributed to this overall failure.

John Steinbeck got the idea for the title of his book, Of Mice and Men, from a poem by Robert Burns entitled To a Mouse. In this poem, Burns refers to the best laid plans of mice and men... and how often those plans run aground. If our hypothesis is correctthat endogenous DHEA acts as a kill switch to prevent tumorigenesis in cells that have experienced p53 inactivation - then Burns' poem will apply to a dishearteningly large segment of research conducted in rodents over the past 40 years.

\section{References}

1. Miller WL. Androgen synthesis in adrenarche. Rev Endocr Metab Disord 2009;10:3-17.

2. Labrie F, Martel C, Belanger A, Pelletier G. Androgens in women are essentially made from DHEA in each peripheral tissue according to intracrinology. J Steroid Biochem Mol Biol 2017;168:9-18.

3. Parker CR Jr, Grizzle WE, Blevins JK, Hawkes K. Development of adrenal cortical zonation and expression of key elements of adrenal androgen production in the chimpanzee (Pan troglodytes) from birth to adulthood. Mol Cell Endocrinol 2014;387:35-43.

4. Blevins JK, Coxworth JE, Herndon JG, Hawkes K. Brief communication: Adrenal androgens and aging: Female chimpanzees (Pan troglodytes) compared with women. Am J Phys Anthropol 2013;151:643-8.
5. Khorram O, Vu L, Yen SS. Activation of immune function by dehydroepiandrosterone (DHEA) in ageadvanced men. J Gerontol A Biol Sci Med Sci 1997;52:M1-7.

6. McNelis JC, Manolopoulos KN, Gathercole LL, et al. Dehydroepiandrosterone exerts antiglucocorticoid action on human preadipocyte proliferation, differentiation, and glucose uptake. Am J Physiol Endocrinol Metab 2013;305:E1134-44.

7. Buford TW, Willoughby DS. Impact of $\operatorname{DHEA}(\mathrm{S})$ and cortisol on immune function in aging: a brief review. Appl Physiol Nutr Metab 2008;33:429-33.

8. Roberts E, Bologa L, Flood JF, Smith GE. Effects of dehydroepiandrosterone and its sulfate on brain tissue in culture and on memory in mice. Brain Res 1987;406:357-62.

9. Compagnone NA, Mellon SH. Dehydroepiandrosterone: A potential signaling molecule for neocortical organization during development. Proc Natl Acad Sci USA 1998;95:4678-83.

10. Nguyen TV, Gower P, Albaugh MD, et al. The developmental relationship between DHEA and visual attention is mediated by structural plasticity of cortico-amygdalar networks. Psychoneuroendocrinology 2016;70: 122-33.

11. Nguyen T-V, McCracken JT, Ducharme $\mathrm{S}$, et al. Interactive Effects of Dehydroepiandrosterone and Testosterone on Cortical Thickness during Early Brain Development. J Neurosci 2013;33:10840-8.

12. Labrie F, Labrie C. DHEA and intracrinology at menopause, a positive choice for evolution of the human species. Climacteric 2013;16:205-13.

13. FDA approves Intrarosa for postmenopausal women experiencing pain during sex. Available from: https://www.fda.gov/newsevents/newsroom/pressannouncements/ucm529641. $\mathrm{htm}$

14. Labrie F, Bélanger A, Pelletier G, et al. Science of intracrinology in postmenopausal women. Menopause 2017;24:702-12.

15. Labrie F, Martel C. A low dose (6.5 mg) of intravaginal DHEA permits a strictly local action while maintaining all serum estrogens or androgens as well as their metabolites within normal values. Horm Mol Biol Clin Investig 2017;29:39-60.

16. Zhang $\mathrm{HH}, \mathrm{Xu} \mathrm{PY}, \mathrm{Wu} \mathrm{J}$, et al. Dehydroepiandrosterone improves follicular fluid bone morphogenetic protein-15 and accumulated embryo score of infertility patients with diminished ovarian reserve undergoing in vitro fertilization: a randomized controlled trial. J Ovarian Res 2014;7:93.

17. Yeung TW, Chai J, Li RH, et al. A randomized, controlled, pilot trial on the effect of dehydroepiandrosterone on ovarian response markers, ovarian response, and in vitro fertilization outcomes in poor responders. Fertil Steril 2014;102:108-15.

18. Ohana D, Maayan R, Delayahu Y, et al. Effect of dehydroepiandrosterone addon therapy on mood, decision making and subsequent relapse of polydrug. Addict Biol 2014;21:885-94.

19. Hough CM, Lindqvist D, Epel ES, et al. Higher serum DHEA concentrations before and after SSRI treatment are associated with remission of major depression. Psychoneuroendocrinology 2017;77:122-30.

20. Schmidt PJ, Daly RC, Bloch M, et al. Dehydroepiandrosterone monotherapy in midlife-onset major and minor depression. Arch Gen Psychiatry 2005;62:154-62.

21. Dumas de La Roque E, Savineau JP, Metivier AC, et al. Dehydroepiandrosterone (DHEA) improves pulmonary hypertension in chronic obstructive pulmonary disease (COPD): a pilot study. Ann Endocrinol (Paris) 2012; 73:20-5.

22. Kuo W-Y, Lin J-Y, Tang K. Human glucose-6-phosphate dehydrogenase (G6PD) gene transforms NIH 3T3 cells and induces tumors in nude mice. Int $\mathrm{J}$ Cancer 2000;85:857-64.

23. Jiang $\mathrm{P}, \mathrm{Du} \mathrm{W}, \mathrm{Wu} \mathrm{M}$. Regulation of the pentose phosphate pathway in cancer. Protein Cell 2014;5:592-602.

24. Jiang $P, D u$ W, Wang X, et al. p53 regulates biosynthesis through direct inactivation of glucose-6-phosphate dehydrogenase. Nat Cell Biol 2011;13:310-6.

25. Cornish-Bowden A. Why is uncompetitive inhibition so rare? A possible explanation, with implications for the design of drug and pesticides. FEBS 1986;203:3-6.

26. Schwartzenberg-Bar-Yoseph F, Armoni M, Karnieli E. The tumor suppressor p53 down- regulates glucose transporters GLUT1 and GLUT4 gene expression. Cancer Res 2004;64:262733.

27. Peschiaroli A, Giacobbe A, Formosa A, et al. miR-143 regulates hexokinase 2 expression in cancer cells. Oncogene 2013;32:797-802.

28. Mayer D, Reuter S, Hoffmann H, et al. Dehydroepiandrosterone reduces expression of glycolytic and gluco- 
neogenic enzymes in the liver of male and female rats. Int $\mathrm{J}$ Oncol 1996;8:1069-78.

29. Murphy SH, Suzuki K, Downes M, et al. Tumor suppressor protein (p)53 is a regulator of NF- $\kappa B$ repression by the glucocorticoid receptor. Proc Natl Acad Sci USA 2011;108:17117-22.

30. Son D-S, Kabir SM, Dong YL, et al. Inhibitory Effect of Tumor Suppressor p53 on Proinflammatory Chemokine Expression in Ovarian Cancer Cells by Reducing Proteasomal Degradation of IкB. PLoS One 2012;7:e51116.

31. Dias NJ, Selcer KW. Steroid sulfatase in the human MG-63 pre-osteoblastic cell line: Antagonistic regulation by glucocorticoids and NFKB. Mol Cell Endocrinol 2016;420:85-96.

32. Sato K, Lemitsu M, Aizawa K, Ajsaka R. Testosterone and DHEA activate the glucose metabolism-related signaling pathway in skeletal muscle. Am J Physiol Endocriol Metabol 2008;294:E961-E68.

33. Yamashita R, Saito T, Satoh S, et al. Effects of Dehydroepiandrosterone on gluconeopgenic enzymes and glucose uptake in human hepatoma cell line, HepG2. Endocr J 2005;52:727-33.

34. Mueller JW, Gilligan LC, Idkowiak J, et al. The regulation of steroid action by sulfation and desulfation. Endocrinol Rev 2015;36:526-63.

35. Warren JC, French AP. Distribution of steroid sulfatase in human tissues. J Clin Endocrinol Metab 1965;25:27882.

36. Walther A, Philipp M, Lozza N, Ehlert $\mathrm{U}$. The rate of change in declining steroid hormones: a new parameter of healthy aging in men? Oncotarget 2916;7:60844-857.

37. Stocking JJ, Fiandalo MV, Pop EA, et al. Characterization of Prostate Cancer in a Functional Eunuch. J Natl Compr Canc Netw 2016;14:1054-60.

38. Campbell B. Adrenarche and the evolution of human life history. Am J Hum Biol 2006;18:569-89.

39. Rutkowski K, Sowa P, RutkowskaTalipska J, et al. Dehydroepiandrosterone (DHEA): hypes and hopes. Drugs 2014;74:1195-207.

40. Bair SR, Mellon SH. Deletion of the Mouse P450c17 Gene Causes Early Embryonic Lethality. Mol Cell Biol 2004;24:5383-90.

41. Schwartz AG, Pashko LL. Mechanism of cancer preventive action of DHEA. Role of glucose-6-phosphate dehydrogenase. Ann NY Acad Sci 1995;774: 180-6.

42. Hursting SD, Perkins SN, Haines DC,
Ward JM, Phang JM. Chemoprevention of spontaneous tumorigenesis in p53knockout mice. Cancer Res 1995;55:3949-53.

43. Schilling MM, Oeser JK, Chandy JK, et al. Sequence variation between the mouse and human Glucose-6Phosphatase Catalytic Subunit gene promoters results in differential activation by Peroxisome Proliferator Activator Receptor Gamma Coactivator-1 $\alpha$. Diabetologia 2008;51: 1505-14.

44. Yokokawa T, Sato K, Iwanaka N, et al. Dehydroepiandrosterone activates AMP kinase and regulate GLUT4 and PGC$1 \alpha$ expression. Biochem Biophys Res Commun 2015;463:42-7.

45. Peto R. Epidemiology, multistage models, and short-term mutagenicity tests. Available

from: w w w. d c s c i e n c e.net/ P e t o $1977 \% 20 \mathrm{CSH} \% 20+\% 20$ proof $\% 20$ corrections.pdf

46. Noble R, Kaltz O, Michael E, Hochberg ME. Peto's paradox and human cancers. Philos Trans R Soc Lond B Biol Sci 2015;370:1-9.

47. Caulin AF, Graham TA, Wang LS, Maley CC. Solutions to Peto's paradox revealed by mathematical modelling and cross-species cancer gene analysis. Philos Trans R Soc Lond B Biol Sci 2015;370:1-7.

48. Sulak M, Fong L, Mika K, et al. TP53 copy number expansion is associated with the evolution of increased body size and an enhanced DNA damage response in elephants. Elife 2016;5:e11994.

49. Schiebinger RJ, Albertson BD, Barnes $\mathrm{KM}$, et al. Developmental changes in rabbit and dog adrenal function: a possible homologue of adrenarche in the dog. Am J Physiol 1981;240:E694-9.

50. Cutler GB Jr, Glenn M, Bush M, et al. Adrenarche: a survey of rodents, domestic animals, and primates. Endocrinology 1978;103:2112-8.

51. Frank LA, Rohrbach BW, Bailey EM, et al. Steroid hormone concentration profiles in healthy intact and neutered dogs before and after cosyntropin administration. Domest Anim Endocrinol 2003; 24:43-57.

52. Odell WD, Parker LN. Control of adrenal androgen production. Endocr Res 1984-85;10:617-30.

53 Perez-Fernandez R, Facchinetti F, Beiras A, et al. Morphological and functional stimulation of adrenal reticularis zone by dopaminergic blockade in dogs. $\mathrm{J}$ Steroid Biochem 1987;28:465-70.

54. van der Molen HJ, Eik-Nes KB.
Biosynthesis and secretion of steroids by the canine testis. Biochim Biophys Acta 1971;248:343-62.

55. Bondarenko LA. Fractional content of androgens in the urine of young sexually mature, old and castrated dogs. Probl Endokrinol (Mosk) 1976;22:81-3.

56. de la Riva GT, Hart BL, Farver TB, et al. Neutering Dogs: Effects on Joint Disorders and Cancers in Golden Retrievers. PLoS One 2013;8:e55937.

57. Nyce J. Method of replenishing steroid hormones in neutered mammals comprising administration of dehydroepiandrosterone (DHEA) and specific metabolites in order to increase steroid hormone levels from those of neutered mammals associated with high cancer risk, to steroid hormone levels of gonadally-intact mammals associated with reduced cancer risk, without inducing the side effects that would occur without metabolite supplementation. US 20170007620 A1, 2015. Available from: http://www.freepatentsonline.com/y2017/0007620.html

58. Sa G, Xiong X, Ren J, et al. Expression of fibrosis-related molecules in the oral mucosa of six animal species: A reference for selecting animal models. Eur J Pharm Sci 2017;96:472-8.

59. Zhang S. Natural history of mevalonate kinase deficiency: a literature review. Pediatr Rheumatol Online J 2016; 14:30.

60. Nyce J. Autoinflammatory reaction in dogs treated for cancer via G6PD inhibition. Case Reports in Veterinary Med 2017;2017:4275305.

61. Durel CA, Aouba A, Bienvenu B, et al. Observational Study of a French and Belgian Multicenter Cohort of 23 Patients Diagnosed in Adulthood With Mevalonate Kinase Deficiency. Medicine (Baltimore) 2016;95:e3027.

62. Schulz S, Klann RC, Schönfeld S, Nyce JW. Mechanisms of cell growth inhibition and cell cycle arrest in human colonic adenocarcinoma cells by dehydroepiandrosterone: role of isoprenoid biosynthesis. Cancer Res 1992;52: 1372-6.

63. Schulz S, Nyce JW. Inhibition of protein isoprenylation and p21ras membrane association by dehydroepiandrosterone in human colonic adenocarcinoma cells in vitro. Cancer Res 1991;51:6563-7.

64. Jurczyluk J, Munoz MA, Skinner OP, et al. Mevalonate kinase deficiency leads to decreased prenylation of Rab GTPases. Immunol Cell Biol 2016;94: 994-9.

65. Marcuzzi A, Crovella S, Pontillo A. Geraniol rescues inflammation in cellu- 
lar and animal models of mevalonate kinase deficiency. In Vivo 2011;25:8792.

66. Marcuzzi A, Pontillo A, De Leo L, et al. Natural isoprenoids are able to reduce inflammation in a mouse model of mevalonate kinase deficiency. Pediatr Res 2008;64:177-82.

67. Kaplan EL, Meier, P. Nonparametric estimation from incomplete observations. J Amer Statist Assn 1958;53:45781.

68. Yamamoto S, Hoshi K, Hirakawa A, et al. Epidemiological, clinical and pathological features of primary cardiac hemangiosarcoma in dogs: a review of 51 cases. J Vet Med Sci 2013;75:143341.

69. Bailey J, Shaw A, Fischer R, et al. A novel role for endothelial tetrahydrobiopterin in mitochondrial redox balance. Free Radic Biol Med 2017;104: 214-25.

70. Zapata E, Ventura JL, De la Cruz K, et al. Dehydroepiandrsterone inhibits the proliferation of human umbilical vein endothelial cells by enh2ncing the expression of $\mathrm{p} 53$ and $\mathrm{p} 21$, restricting the phosphorylation of retinoblastoma protein, and is androgen- and estrogenreceptor independent. FEBS J 2005;272:1343-53.

71. Calvert AE, Chalastanis A, Wu Y, et al. Cancer-Associated IDH1 Promotes Growth and Resistance to Targeted Therapies in the Absence of Mutation. Cell Rep 2017;19:1858-73.

72. Van Damme M, Crompot E, Meuleman $\mathrm{N}$, et al. Characterization of TET and IDH gene expression in chronic lymphocytic leukemia: comparison with normal B cells and prognostic significance. Clin Epigenetics 2016;8:132.

73. Zhang $\mathrm{C}$, Moore LM, Li X, et al. IDH1/2 mutations target a key hallmark of cancer by deregulating cellular metabolism in glioma. Neuro Oncol 2013;15:1114-26.

74. Leduc C, Jenkins SM, Sukov WR, et al. Cardiac angiosarcoma: histopathologic, immunohistochemical, and cytogenetic analysis of 10 cases. Hum Pathol 2017;60:199-207.

75. Dang L, Yen K, Attar EC, et al. IDH mutations in cancer and progress toward development of targeted therapeutics. Ann Oncol 2016;27:599-608.

76. Flöter J, Kaymak I, Schulze A. Regulation of Metabolic Activity by p53. Metabolites 2017;7:E21.

77. Mukherjee, Siddhartha, The Emperor of All Maladies: A Biography of Cancer. Simon and Schuster, New York, 2010.

78. Erridge SC, Murray B, Price A, et al. Improved Treatment and Survival for Lung Cancer Patients in South-East Scotland. J Thoracic Oncol 2008;3:4918.

79. Cancer Research UK. Lung cancer statistics. Available from: http://www.cancerresearchuk.org/health-professional/ cancer-statistics/statistics-by-cancertype/lung-cancer\#heading-Two

80. Iafolla MAJ, Juergens RA. Update on Programmed Death-1 and Programmed Death-Ligand 1 Inhibition in the Treatment of Advanced or Metastatic Non-Small Cell Lung Cancer. Front Oncol 2017;7:67. 